Supporting Information for:

\title{
Particle-phase diffusion modulates partitioning of semivolatile organic compounds to aged secondary organic aerosol
}

Rahul A. Zaveri, ${ }^{1, *}$ John E. Shilling, ${ }^{1}$ Alla Zelenyuk, ${ }^{1}$ Maria A. Zawadowicz, ${ }^{1}$ Kaitlyn Suski, ${ }^{1, \S}$

Swarup China, ${ }^{2}$ David M. Bell ${ }^{1, \neq}$, Daniel Veghte, ${ }^{2,+}$ and Alexander Laskin ${ }^{3}$

${ }^{1}$ Atmospheric Sciences and Global Change Division, Pacific Northwest National Laboratory, Richland, WA 99352, United States

${ }^{2}$ William R. Wiley Environmental Molecular Sciences Laboratory, Pacific Northwest National Laboratory, Richland, WA 99352, United States

${ }^{3}$ Department of Chemistry, Purdue University, West Lafayette, IN 47907, United States

*Correspondence to: R. A. Zaveri (rahul.zaveri@pnnl.gov)

This file includes:

Pages S1-S8

Materials and Methods

Figures S1-S3

References 


\section{Instruments}

\section{Single particle mass spectrometer miniSPLAT}

4 A single particle mass spectrometer, miniSPLAT, was used for real-time characterization of

5 particle vacuum aerodynamic diameter $\left(D_{v a}\right)$, density, shape, and composition. A detailed

6 description of miniSPLAT and its application to quantify room-temperature evaporation kinetics

7 of size-selected particles are provided elsewhere. ${ }^{1-3}$ Briefly, individual aerosol particles,

8 sampled into miniSPLAT through an aerodynamic lens inlet are detected by light scattering in

9 two optical stages $10.9 \mathrm{~cm}$ apart. $^{3}$ The particle time-of-flight between the two optical stages

10 yields particle velocity, which is used to calculate particle $D_{v a}$ with precision and accuracy of

$11 \pm 0.5 \%$. The dual particle detection is also used to generate a trigger for the excimer laser, used

12 o generate positive and negative ions and acquire single particle mass spectra. Measurements

13 conducted on size- or mass-selected particles provide information on particle shape, precise

14 density $( \pm 0.5 \%)$, and evaporation kinetics.

\section{Nano-DESI-HRMS}

17 Off-line filter samples were collected on membrane filters (TE36, Whatman, $0.45 \mu \mathrm{m}$ pore size) using a four-stage cascade impactor (Sioutas Personal Cascade Impactor, SKC). Samples were

19 collected on stages $C$ and D (particle cut-off sizes: stage $C-0.50 \mu \mathrm{m}$, and stage $D-0.25 \mu \mathrm{m}$ ).

20 Samples were deposited along impinging jet streamlines on filter surface. In this way instead of

21 samples scattered around the entire filter, particles were deposited along a line. This sampling 
22 method is ideal for probing with the custom-built Nanospray Desorption Electrospray Ionization

23 (Nano-DESI) source coupled to a high resolution LTQ-Orbitrap mass spectrometer (Thermo

24 Electron, Bremen, Germany). Briefly, the sample was placed on a computer-controlled XYZ

25 stage and brought in contact with the Nano-DESI probe, which was assembled using two fused

26 silica capillaries $(50 \times 185 \mu \mathrm{m}$, ID $\times$ OD, Polymicro Technologies, L.L.C., Phoenix). A syringe pump

27 at a flow rate of $0.3 \mu \mathrm{L} \mathrm{min}^{-1}$ was used to infuse the solvent $(70 / 30 \% \text {, acetonitrile/water })^{4}$. The

28 experiments were typically performed in the positive ionization mode with a resolving power of

$29 \mathrm{~m} / \Delta \mathrm{m}=10^{5}$ at $\mathrm{m} / \mathrm{z} 400$, with spray voltage of $3.5 \mathrm{kV}$, and the capillary heated to $250^{\circ} \mathrm{C}$. The

30 instrument was calibrated using a standard calibration MSCAL 5 mixture (Sigma-Aldrich, Inc.)

31 prior to experiments. Mass spectral features with a minimum signal-to-noise ratio of 3 were

32 extracted from the averaged mass spectra of both solvent background and sample using Decon

332 2LS software ${ }^{5}$ (http://omics.pnl.gov/software/decontools-decon2ls). HRMS data were

34 processed using a suite of Microsoft Excel macros, including background subtraction, first and

35 second-order mass defect analysis. ${ }^{6,7}$

\section{Box Model}

38 In this study we used the sectional aerosol box model MOSAIC (Model for Simulating Aerosol

39 Interactions and Chemistry $)^{8,9}$ to interpret the observed aerosol growth kinetics. MOSAIC

40 dynamically partitions multiple compounds to all particle size bins (mean diameter, $D_{p}$ ) by

41 taking into account compound volatility $\left(C^{*}\right)$, gas-phase diffusion $\left(D_{g}\right)$, interfacial mass

42 accommodation $(\alpha)$, intra-particle bulk diffusion $\left(D_{b}\right)$, and reversible particle-phase reactions. In

43 this study, $D_{\mathrm{g}}$ was assumed at $0.05 \mathrm{~cm}^{2} \mathrm{~s}^{-1}$ and $\alpha=0.1$ for all condensing compounds. The 
44 thermodynamic driving force for mass transfer is based on Raoult's law. Bulk diffusion is treated

45 using a combination of (1) an analytical quasi-steady-state treatment for the diffusion-reaction

46 process within the particle phase for fast-reacting organic compounds such that the timescales

47 for their particle-phase concentrations to reach quasi-steady state are shorter than $1 \mathrm{~min}$, and

48 (2) a two-film theory approach for slow- and non-reacting organic compounds. The

49 logarithmically spaced bin structure in the MOSAIC was same as that of the particle size

50 distribution data provided by the SMPS. The number of size bins in the model was set at 120.

51 Transfer of particles between bins due to growth or shrinkage was calculated using a two-

52 moment approach. Coagulation was accounted for in the simulations. 

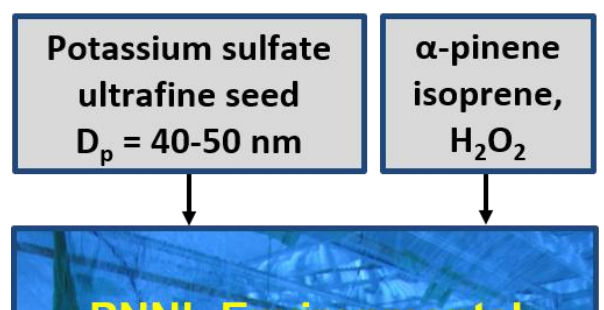

PNNL Environmental

Chamber $\left(10.6 \mathrm{~m}^{3}\right)$
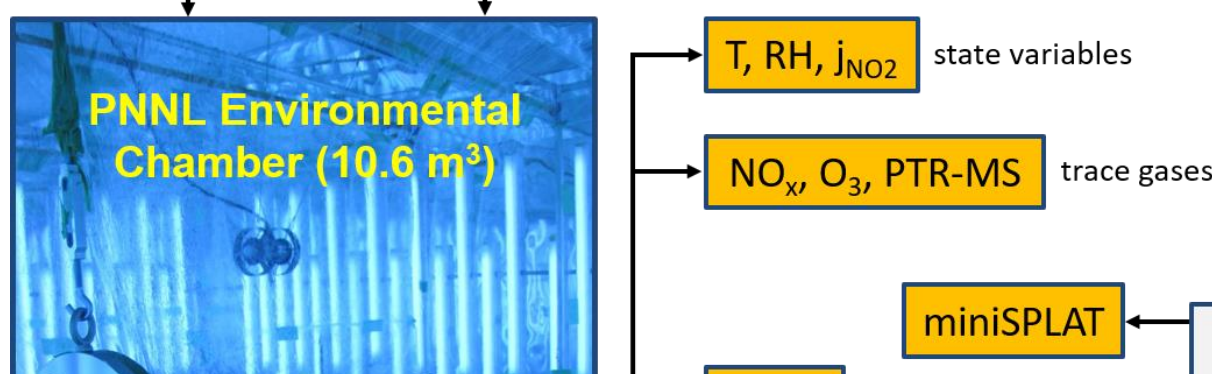

(2)

DMA

size-selected
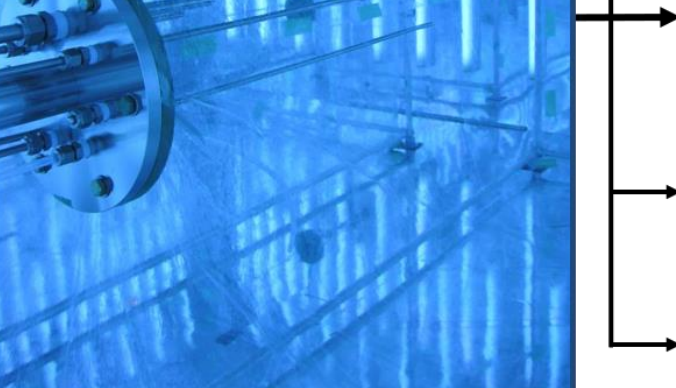

SMPS

aerosol size distribution

2" layer of activated charcoal

Figure S1. Schematic of experiment set up. Aerosol growth kinetics experiments were conducted in $10.6 \mathrm{~m}^{3}$ FEP Teflon environmental chamber at Pacific Northwest National Laboratory. The chamber was run in the so-called batch mode where reactants were added to the chamber in discrete quantities. Instruments shown with orange background measured in real time (i.e., online) the state variables, trace gases, particle size distribution. Filter samples were collected at the end of the experiment for offline chemical analysis. Size-selected SOA particles formed in the environmental chamber were transferred at various stages of the experiment into separate small chambers to study their evaporation kinetics at room temperature under dry $(\mathrm{RH}<5 \%)$ and humid $(\mathrm{RH}=75 \%)$ conditions. 

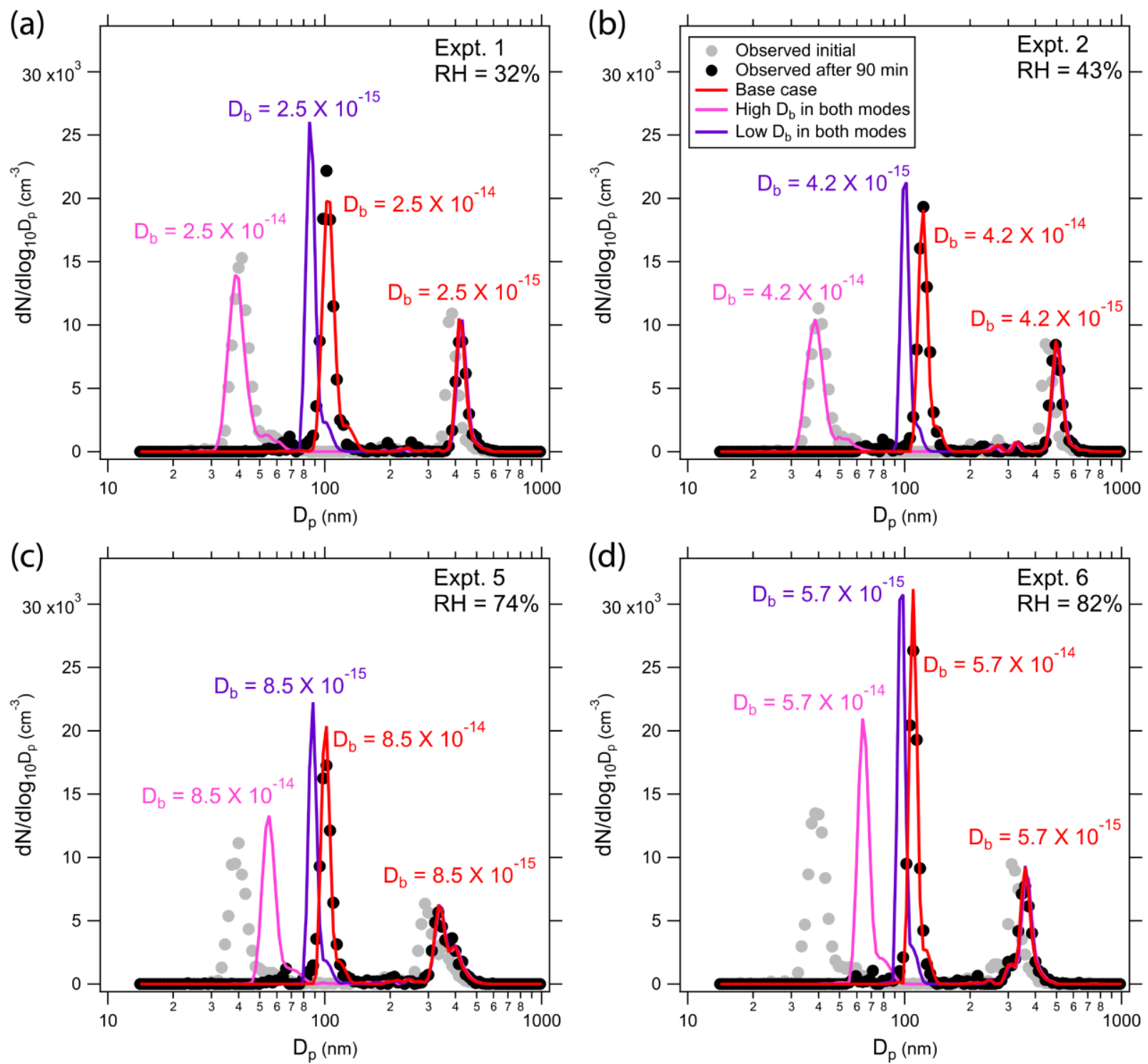

65

Figure S2. Sensitivity of growth kinetics to the prescribed bulk diffusivity values $\left(\mathrm{cm}^{2} \mathrm{~s}^{-1}\right)$ for the Aitken and accumulation modes. The base case $D_{b}$ values (red) are the same as the semisolid scenario shown in Figure 4. In the "high $D_{b}$ " case, the bulk diffusivity for both aerosol modes is set to the value shown in pink. In the "low $D_{b}$ " case, the bulk diffusivity for both aerosol modes is set to the value shown in purple. 

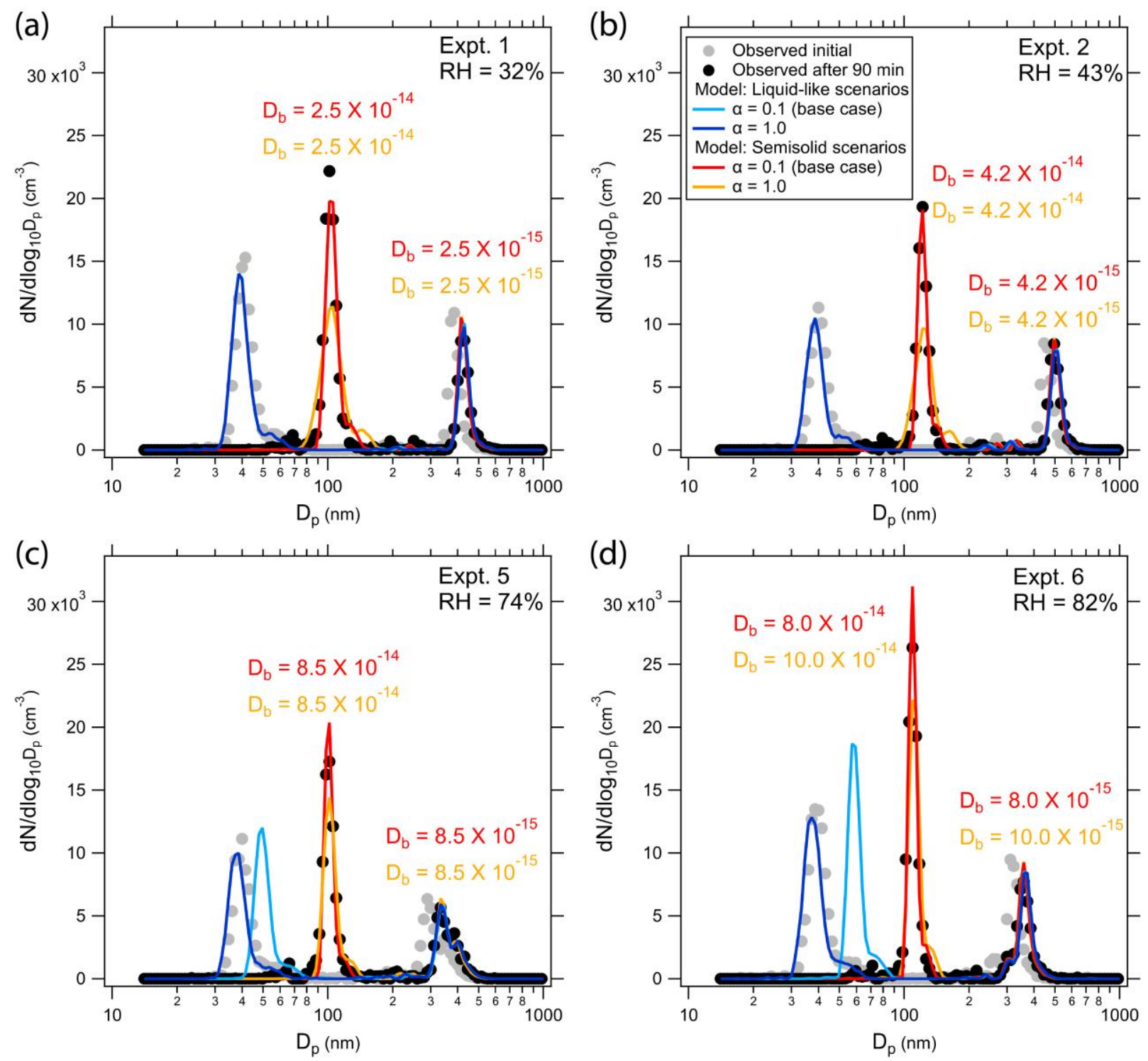

74 Figure S3. Sensitivity of growth kinetics to the prescribed mass accommodation $(\alpha)$ values for 75 both the Aitken and accumulation modes, assuming isoprene and a-pinene SOA in the accumulation mode are miscible in both liquid-like and semisolid scenarios. The base case scenarios are the same as those shown in Figure 4. The liquid-like scenarios assume $D_{b}>10^{-10}$ $\mathrm{cm}^{2} \mathrm{~s}^{-1}$. The $D_{b}$ values required for the two aerosol modes in the semisolid scenarios are indicated in each panel; the values in red are for the base case $(\alpha=0.1)$ and the values in orange are for $\alpha=0.1$. 
References

84 1. Vaden, T. D.; Imre, D.; Beranek, J.; Shrivastava, M.; Zelenyuk, A., Evaporation kinetics and phase 85 of laboratory and ambient secondary organic aerosol. Proceedings of the National Academy of Sciences of 86 the United States of America 2011, 108, (6), 2190-2195.

87 2. Wilson, J.; Imre, D.; Beránek, J.; Shrivastava, M.; Zelenyuk, A., Evaporation Kinetics of Laboratory88 Generated Secondary Organic Aerosols at Elevated Relative Humidity. Environmental Science \& 89 Technology 2015, 49, (1), 243-249.

$90 \quad 3 . \quad$ Zelenyuk, A.; Imre, D.; Wilson, J.; Zhang, Z.; Wang, J.; Mueller, K., Airborne Single Particle Mass 91 Spectrometers (SPLAT II \& miniSPLAT) and New Software for Data Visualization and Analysis in a Geo92 Spatial Context. Journal of The American Society for Mass Spectrometry 2015, 26, (2), 257-270.

93 4. Roach, P. J.; Laskin, J.; Laskin, A., Nanospray desorption electrospray ionization: an ambient 94 method for liquid-extraction surface sampling in mass spectrometry. Analyst 2010, 135, (9), 2233-2236.

95 5. Jaitly, N.; Mayampurath, A.; Littlefield, K.; Adkins, J. N.; Anderson, G. A.; Smith, R. D., Decon2LS: 96 An open-source software package for automated processing and visualization of high resolution mass 97 spectrometry data. BMC Bioinformatics 2009, 10, 87.

$98 \quad 6 . \quad$ Eckert, P. A.; Roach, P. J.; Laskin, A.; Laskin, J., Chemical characterization of crude petroleum using 99 nanospray desorption electrospray ionization coupled with high-resolution mass spectrometry. Anal Chem 2012, 84, (3), 1517-25.

$1017 . \quad$ Roach, P. J.; Laskin, J.; Laskin, A., Higher-Order Mass Defect Analysis for Mass Spectra of Complex 102 Organic Mixtures. Analytical Chemistry 2011, 83, (12), 4924-4929.

103 8. Zaveri, R. A.; Easter, R. C.; Fast, J. D.; Peters, L. K., Model for Simulating Aerosol Interactions and 104 Chemistry (MOSAIC). Journal of Geophysical Research-Atmospheres 2008, 113, D13204.

105 9. Zaveri, R. A.; Easter, R. C.; Shilling, J. E.; Seinfeld, J. H., Modeling kinetic partitioning of secondary 106 organic aerosol and size distribution dynamics: representing effects of volatility, phase state, and particle107 phase reaction. Atmospheric Chemistry and Physics 2014, 14, (10), 5153-5181. 